\title{
Solar wind sputtering of dust on the surface of 67P/Churyumov-Gerasimenko
}

\author{
Peter Wurz ${ }^{1,2}$, Martin Rubin ${ }^{1}$, Kathrin Altwegg ${ }^{1,2}$, Hans Balsiger ${ }^{1}$, Jean-Jacques Berthelier ${ }^{3}$, André Bieler ${ }^{4}$ \\ Ursina Calmonte $^{1}$, Johan De Keyser ${ }^{5}$, Björn Fiethe ${ }^{6}$, Stephen A. Fuselier ${ }^{7,8}$, André Galli ${ }^{1}$, Sébastien Gasc ${ }^{1}$, \\ Tamas I. Gombosi ${ }^{4}$, Annette Jäckel ${ }^{1}$, Léna Le Roy ${ }^{1}$, Urs A. Mall ${ }^{9}$, Henri Rème ${ }^{10,11}$, \\ Valeriy Tenishev ${ }^{4}$, and Chia-Yu Tzou ${ }^{1}$ \\ 1 Physics Institute, University of Bern, Sidlerstrasse 5, 3012 Bern, Switzerland \\ e-mail: peter.wurz@space.unibe.ch \\ 2 Center for Space and Habitability (CSH), University of Bern, 3012 Bern, Switzerland \\ 3 LATMOS/IPSL/CNRS-UPMC-UVSQ, 4 place Jussieu, 75252 Paris Cedex 05, France \\ ${ }^{4}$ University of Michigan, Ann Arbor, MI 48109, USA \\ 5 Belgian Institute for Space Aeronomy, 1180 Brussels, Belgium \\ 6 Technical University of Braunschweig, 38106 Braunschweig, Germany \\ 7 Southwest Research Institute, San Antonio, TX 78228-0510, USA \\ 8 University of Texas at San Antonio, San Antonio, TX 78249, USA \\ 9 Max Planck Institute for Solar System Research, 37077 Göttingen, Germany \\ ${ }^{10}$ University of Toulouse, UPS-OMP, IRAP, 31000 Toulouse, France \\ 11 CNRS, IRAP, Toulouse Cedex 4, 31055 Toulouse, France
}

Received 27 February 2015 / Accepted 27 May 2015

\section{ABSTRACT}

\begin{abstract}
Aims. Far away from the Sun, at around $3 \mathrm{AU}$, the activity of comet 67P/Churyumov-Gerasimenko is low and changes with local time (solar insolation), with location (chemical heterogeneity of the surface), and with season. When the activity is very low because the total cross section of the comet against the Sun is small, the solar wind has access to the surface of the comet and causes ion-induced sputtering of surface material, which we wish to observe.

Methods. We used the Double Focussing Mass Spectrometer (DFMS) of the ROSINA experiment on ESA's Rosetta mission to search for mass spectrometric evidence of sputtered refractory species. In high-resolution mode, DFMS can separate some of the mass peaks of refractory elements from the many volatile species present in the coma.

Results. At present, the locations of solar wind surface access are in the southern hemisphere of the comet (the local winter). Of particular interest is sputtering of dust grains on the surface. We observe global averages over the winter hemisphere of the refractory elements $\mathrm{Na}, \mathrm{K}, \mathrm{Si}$, and $\mathrm{Ca}$, presumably sputtered from grains residing on the surface. Compared to carbonaceous chondrites, the comet has the same $\mathrm{Na}$ abundance, is depleted in $\mathrm{Ca}$, and has an excess of $\mathrm{K}$. In addition, for Si the signal strength is strong enough to compile a coarse compositional map of the southern hemisphere. Most, perhaps all, of the observed variation can be explained by the solar wind being affected by the atmosphere of the comet.
\end{abstract}

Key words. comets: individual: 67P/Churyumov-Gerasimenko

\section{Introduction}

While the Rosetta spacecraft of the European Space Agency (ESA) was orbiting close to comet 67P/ChuryumovGerasimenko (67P), we performed continuous measurements of the chemical inventory of its coma with the Rosetta Orbiter Spectrometer for Ion and Neutral Analysis (ROSINA) instrument suite. ROSINA consists of two mass spectrometers, the Double Focusing Mass Spectrometer (DFMS) and the Reflectron-type Time-Of-Flight (RTOF), as well as the ROSINA COmet Pressure Sensor (COPS; Balsiger et al. 2007). Most of the observed species in the coma are volatile material that are released from the comet's surface by sublimation, for example, $\mathrm{H}_{2} \mathrm{O}, \mathrm{CO}, \mathrm{CO}_{2}$ (Hässig et al. 2015), and many others (Le Roy et al. 2015). The number densities in the coma of the volatile species show temporary variations that are controlled by solar illumination (diurnal cycle) with the rotation period of the comet of $12.4 \mathrm{~h}$ and half of that, with seasonal variation (summer and winter hemispheres), and also compositional heterogeneity of the volatile inventory on the cometary surface. We normalize the measurements of the observed species onto a spherical surface of $10 \mathrm{~km}$ from the center of the nucleus to create coarse maps of their probable origin on the surface.

In addition to the volatile material, we detected atoms of $\mathrm{C}$, $\mathrm{O}, \mathrm{Na}, \mathrm{K}, \mathrm{Si}, \mathrm{Ca}$, and $\mathrm{S}$, which can at best only be partially set free through sublimation or are fragments of sublimed molecules (Le Roy et al. 2015). For the species that are not dominated by fragments from volatile species, we also create these maps of possible origin. The maps for the sputtered atoms differ significantly from the maps for volatile species, such as the water map, and in some cases they are almost anticorrelated. Our present understanding is that these atoms are the result of solar 
wind sputtering of refractory material, that is, of dust located on the cometary surface. Since the release of material from the surface by sputtering can be calculated by the TRIM software (Ziegler et al. 1984; Ziegler 2004), we can infer the chemical composition of major elements for the areas affected by sputtering, and the average mineralogy of these locations may be derived. The sputter signal from the comet will disappear as the comet approaches the Sun and becomes more active. Once the coma is dense enough, the solar wind will be absorbed by the gas layer above the surface and will no longer propagate to the surface, and after the formation of a diamagnetic cavity, the solar wind will be deflected around the comet. For the periods of high density during current conditions, the distance of this cavity from the cometary surface is estimated to be in the range of 10-20 km (calculated with Eq. (10.30) in Gombosi 2015).

\section{DFMS instrument}

We only use the DFMS instrument for these observations because it has the necessary mass resolution to separate the peaks of the refractory species from the much more abundant volatile species. DFMS has two operational modes, an ion mode and a neutral gas mode. The ion mode has been used occasionally, but not within $20 \mathrm{~km}$ from the comet (Fuselier et al. 2015). Since there are no ion mode data closer than $20 \mathrm{~km}$ altitude, we concentrate on the gas mode observations. DFMS has a mass resolution of $m / \Delta m=3000$ at the $1 \%$ peak level at mass $28 \mathrm{u} / \mathrm{e}$ (corresponding to $m / \Delta m \approx 9000$ at the $50 \%$ peak level). DFMS uses a combination of an electrostatic and a magnetic sector analyzer, and by choosing a suitable set of voltages, ions with a chosen ratio of mass per charge are transmitted through the analyzer system onto a position-sensitive microchannel plate (MCP) detector (Balsiger et al. 2007).

Neutral gas from the environment of the spacecraft enters the DFMS ion source and is ionized there through electron impact ionization. Electrons are emitted by a Tungsten-Rhenium filament operated at $200 \mu \mathrm{A}$ emission and are then accelerated to $45 \mathrm{eV}$ before ionizing the neutral atoms and molecules in the ion source. The positively charged ions are extracted from the ion source and subsequently accelerated into the mass analyzer section.

Only ions with suitable energy are able to pass the $90^{\circ}$ electrostatic analyzer, while only ions with the proper momentum are deflected in the magnetic field by the required angle of $60^{\circ}$ to reach the detector assembly. The selection of ions with a particular momentum and energy can be converted into mass per charge. Details of the functioning of DFMS are given in Balsiger et al. (2007). Each mass per charge is therefore scanned individually and in sequential order. It takes roughly $20 \mathrm{~s}$ per spectrum, and a scan through the full mass range takes approximately $45 \mathrm{~min}$. This also implies that species with different mass per charge are obtained at different times. To obtain ratios between the observed species, we therefore need to interpolate the observations in time, or perform large area averages.

The nominal field of view of the DFMS instrument is $20^{\circ} \times$ $20^{\circ}$ (Balsiger et al. 2007) and effectively is even wider, thus encompassing the entire comet at distances of $>10 \mathrm{~km}$.

\section{Data analysis}

\subsection{Observation scenario}

The time period we investigated to derive the sputtered signal is from 17-24 October 2014. The reasons for choosing

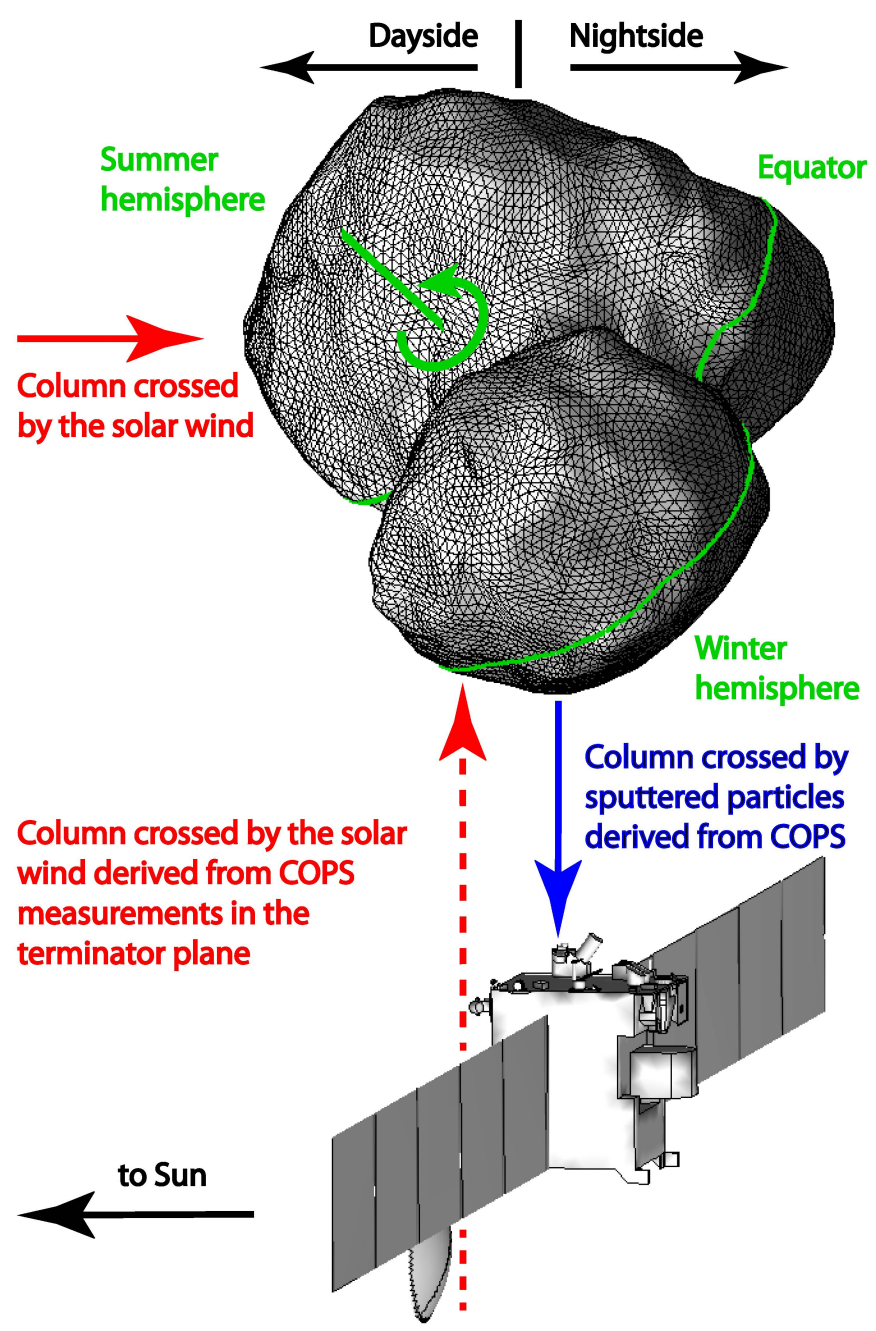

Fig. 1. Geometry of the observation of the solar wind sputtering signal. Rosetta orbits the comet in the terminator plane, at roughly $10 \mathrm{~km}$ from the center of the comet. Shape model from Jorda et al. (2015).

this time period are that the spacecraft was close to the comet, that is, within about $10 \mathrm{~km}$ from the nucleus. During this period, DFMS was exclusively operated in the neutral gas mode. Furthermore, the spacecraft encountered large variations in the observed volatile densities in the terminator orbit (Hässig et al. 2015), which allows studying the abundance of sputtered atoms and how this abundance varies with the neutral gas column that has to be crossed by the solar wind to reach the surface of the comet.

The Rosetta spacecraft was in bound terminator orbits with altitudes ranging from 9.2 to $10.2 \mathrm{~km}$. Since the sputtered signal is very low, it is important to be as close to the comet as possible. The solar wind, which causes the sputtering of surface material, always arrives from about $90^{\circ}$ away from the viewing direction of DFMS in terminator orbits (red dotted arrow in Fig. 1). For this measurement, the arriving solar wind ions first have to pass the atmosphere of the comet and reach the surface (solid red arrow in Fig. 1), and second, particles sputtered from the surface have to reach the spacecraft (blue arrow in Fig. 1).

\subsection{Data treatment}

This section describes the way all the data were treated. First, the offset on the MCP detector was subtracted, which measures 
the total charge, that is, the number of electrons exiting the individual MCP channels during the 20-s measurement time for a mass line. Second, each of the 512 channels on the MCP detector was corrected for variations in the signal gain (i.e., the flat-field correction). In general, this correction is a function of accumulated lifetime extracted charge on each channel over the time of operation, or in other words, the detector efficiency drops during the lifetime. This correction can be monitored throughout the mission by scanning a peak, for example, water at mass per charge $18 \mathrm{u} / \mathrm{e}$, across the detector and investigating the signal obtained on each of the pixels across the detector.

Furthermore, the signal was corrected for the geometrical cross section of the comet as seen from DFMS. For this purpose, the obtained signal was divided by the cosine of the off-pointing from the nadir direction to the comet.

\subsection{DFMS detection sensitivity}

The DFMS instrument sensitivity for the observed sputtered species is not well known, but with the knowledge of most instrument details, it is possible to derive the approximate relative sensitivities. The detection efficiency of an MCP detector like that of DFMS is proportional to the velocity of the incident particle (Meier \& Eberhardt 1993). Furthermore, the higher the mass of the ion, the lower the energy has to be to pass through the electrostatic and magnetic analyzers. Both the instrument transmission and the detection efficiency are a function of the mass. We established by calibration that the combined effect is a mass dependence of $m^{-0.82}$ (where $m$ is mass of the ion). In addition, the ionization cross section of the different species was considered. We used the cross sections listed in Table 1 to correct for the relative sensitivities. Species with a large ionization cross section are detected more easily by DFMS and therefore have a higher sensitivity than species with smaller cross sections. The ionization cross sections, $\sigma$, of the observed species enters linearly into the relative sensitivity of the observed species. This cross section accounts for the instrument-related relative detection efficiencies. We also included a representative solar wind sputtering yield, $Y$, of the particles from the surface, which were derived by TRIM (Ziegler et al. 1984; Ziegler 2004). This yield of course depends on the actual energy of the incident protons and alpha particles (Wurz 2012), which varies with the solar wind and the specific mineralogy on the surface of the comet. The sputter yield can therefore only be approximated.

The counts, $c_{a}$, registered by DFMS for a gas density $n_{a}$ of species $a$ are

$c_{a}=n_{a} \mathrm{GF} \sigma_{a} / m_{a}{ }^{0.82}$,

with the geometric factor GF of DFMS, the cross section for ionization, $\sigma_{a}$, and the mass $m_{a}$. For the density ratio between species $a$ and $b$ at the spacecraft location it therefore follows that

$R_{a b}=\left.\frac{n_{a}}{n_{b}}\right|_{S / C}=\frac{\sigma_{b}}{\sigma_{a}}\left(\frac{m_{a}}{m_{b}}\right)^{0.82} \frac{c_{a}}{c_{b}}=\frac{f_{a}}{v_{a}} \frac{v_{b}}{f_{b}}$,

where $c_{a}$ is the measured signal of species $a$ detected on the MCP detector, that is, the integral below the peak, as shown in Fig. 2, and the flux and average velocity of the sputtered species are $f_{a}$ and $v_{a}$, respectively, with $f_{a}=f_{S W} Y_{a} C_{a}$ and $f_{b}=f_{S W} Y_{b} C_{b}$, and $Y_{a}$ and $Y_{b}$ are the relative sputter yields for species $a$ and $b$ given in Table 1 , and $C_{a}$ and $C_{b}$ the concentrations on the surface for the two species. $f_{S W}$ is the solar wind ion flux impinging on the cometary surface. The average velocity of sputtered

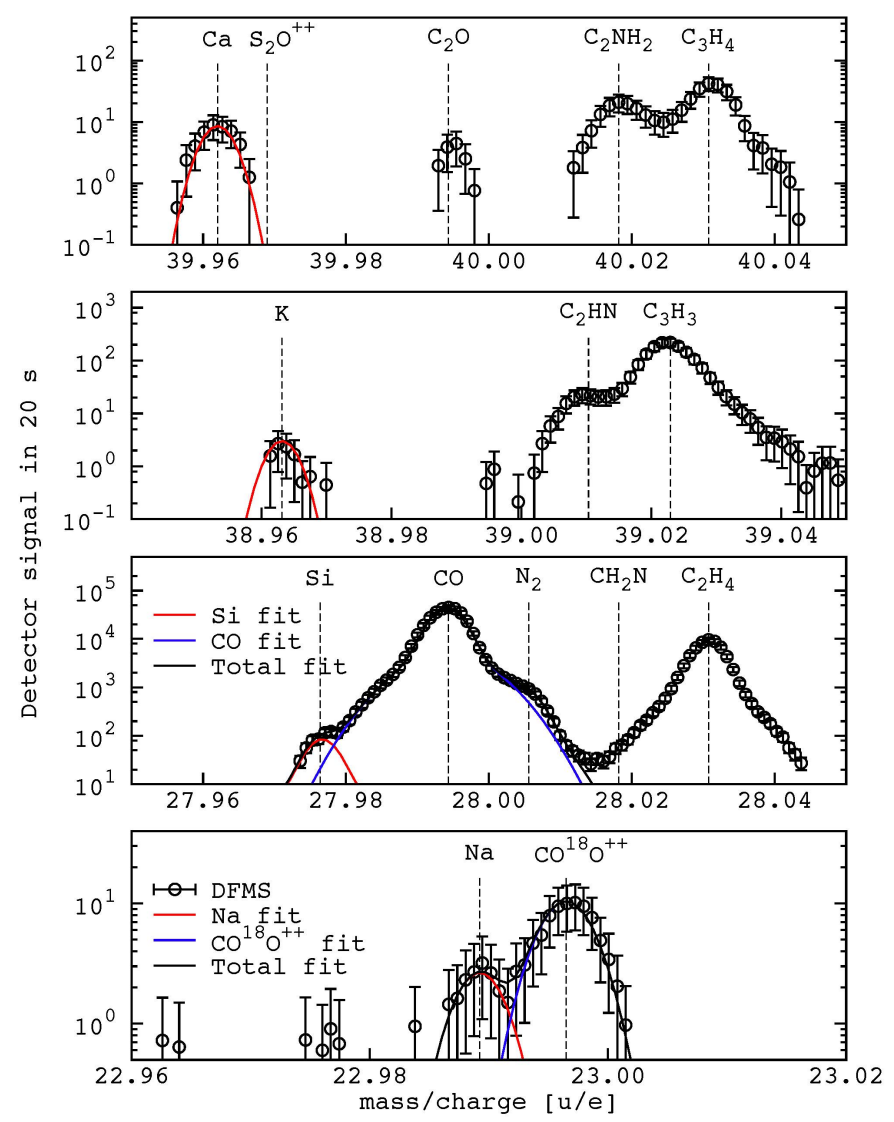

Fig. 2. High-resolution mass spectra from DFMS/ROSINA for the species $\mathrm{Ca}, \mathrm{K}, \mathrm{Si}$ and $\mathrm{Na}$, which are observed together with other species from the volatile material of the coma. Red lines represent Gaussian fits to the mass peaks of interest, blue lines to mass peaks that are close to the mass peak of interest, and the black lines are the sum curve.

atoms is given by the surface binding energy of the atom, $E_{b}$, as $\left.v=\sqrt{(} E_{b} / m\right)$ (Wurz et al. 2007), and the values for the binding energies used are given in Table 1. Equation (2) also neglects the varying amount of protons and alpha particles in the solar wind, but this cancels out when considering element ratios. Since the velocities do not change from the comet to spacecraft because of the negligible gravity of the nucleus, and the fluxes scale reciprocal with the distance squared, Eq. (2) also gives the density ratio at the cometary surface. Thus, we obtain for the ratio of element concentrations, $C_{a}$ and $C_{b}$, on the surface

$\frac{C_{a}}{C_{b}}=\frac{\sigma_{b}}{\sigma_{a}}\left(\frac{m_{a}}{m_{b}}\right)^{0.82} \frac{c_{a}}{c_{b}} \frac{v_{a}}{v_{b}} \frac{Y_{b}}{Y_{a}}$.

Each species was analyzed over several Earth days, to be precise, from 17 October 2014, 12 UT to 24 October 2014, 12 UT over the course of two consecutive $10 \mathrm{~km}$ bound orbits in the terminator plane. This includes more than ten rotations of the nucleus and more than 130 individual measurements of each species (see Fig. 3 top and third panel, which show the individual measurements). Therefore, at least part of the variation in the solar wind density should be cancelled out in the interpolation process. In addition, one mass per charge scan ranging from $23 \mathrm{u} / \mathrm{e}$ to $40 \mathrm{u} / \mathrm{e}$ is obtained within less than $10 \mathrm{~min}$. Long-term variations of the solar wind therefore also vanish when reporting the corresponding ratio of the species. 
Table 1. Mass of the observed species, the corresponding electron impact cross-section, and calculated relative sputtering yield off the surface of the comet.

\begin{tabular}{c|c|c|cl}
\hline \hline Species & Mass/charge & Electron-impact cross section & Relative sputtering yield & Surface binding energy, $E_{b}$ \\
\hline Sodium $(\mathrm{Na})$ & $22.9892 \mathrm{u} / \mathrm{e}$ & $2.6 \AA^{2}$ (Fujii and Srivastava 1995) & 0.197 & $2.0 \mathrm{eV}$ \\
Silicon $(\mathrm{Si})$ & $27.9764 \mathrm{u} / \mathrm{e}$ & $6.5 \AA^{2}$ (Freund et al. 1990) & 0.0613 & $4.7 \mathrm{eV}$ \\
Potassium $(\mathrm{K})$ & $38.9632 \mathrm{u} / \mathrm{e}$ & $5.2 \AA^{2}$ (Borovik 2012) & 0.180 & $0.93 \mathrm{eV}$ \\
Calcium $(\mathrm{Ca})$ & $39.9620 \mathrm{u} / \mathrm{e}$ & $6.5 \AA^{2}$ (Vainshtein et al. 1972) & 0.161 & $2.1 \mathrm{eV}$ \\
\hline
\end{tabular}

Notes. Sputter yields are given for a solar wind velocity of $440 \mathrm{~km} \mathrm{~s}^{-1}$ and a solar wind composition of $95 \% \mathrm{H}$ and $5 \%$ He. The masses have been taken from Haynes (2013) and the mass per charge of the corresponding ion has been calculated by subtracting the mass of the missing electron.

\subsection{Solar wind sputtering}

Incident solar wind protons (and heavier atoms) sputter neutral atoms and molecules off the surface of the comet, but only protons and alpha particles contribute significantly to the sputter yield for regular solar wind (Wurz et al. 2007, 2010; Wurz 2012). The sputter yields for the different species of a grain were obtained using the TRIM.SP calculation (Biersack \& Eckstein 1984; Ziegler et al. 1984; Ziegler 2004), see also the recent review on computer simulation of sputtering by Eckstein \& Urbassek (2007). TRIM provides the total sputter yield for a species and does not specify the fraction of ions in the sputtered flux. TRIM has been used for decades to simulate sputtering and related phenomena and gives good quantitative results on sputter yields, even for samples with relevance to planetology (e.g., Hijazi et al. 2014).

Table 1 lists the calculated relative sputter yields for mineral grains for the species of interest for solar wind speeds of $440 \mathrm{~km} \mathrm{~s}^{-1}$ and assuming 95\% protons and 5\% alpha particles. The He ions contribute about $30 \%$ to the sputter yield. By coincidence, the yield for solar wind sputtering of mineral grains peaks for the typical solar wind ion energies around a specific energy of about $1 \mathrm{keV} \mathrm{nuc}^{-1}$, with a rather flat velocity dependence within the typical the solar wind velocity range (Wehner et al. 1963; Wurz et al. 2007; Wurz 2012). Relative sputter yield means that if an atom on the surface is hit by a solar wind ion, it will be released from the surface with that probability. The absolute sputter yield also considers the abundance of a species on the surface for the sputter yield (Wurz et al. 2007, 2010).

The sputtered atoms are released from the surface with a cosine angular distribution centered at the local surface normal of the facet hit by the solar wind (Wurz et al. 2007, 2010). Thus, despite the $90^{\circ}$ angle between ion impact on the comet and the Rosetta nadir direction, we expect a sputtered signal at the location of the spacecraft. Moreover, the comet does not have a spherical shape, and there are always areas with a surface orientation in between the solar wind arrival direction and the Rosetta observation direction.

The solar wind speed at the spacecraft was $390 \mathrm{~km} \mathrm{~s}^{-1}$ on 20 October 2014 (Broiles et al. 2015) and $370 \mathrm{~km} \mathrm{~s}^{-1}$ on 30 October 2014 (Fuselier et al. 2015), which is indicative of regular slow solar wind during this time period.

We only considered sputtered neutral atoms in this analysis. However, it is known that atomic and molecular ions are also sputtered from solids, with ion yields ranging from $10^{-5}$ to 1 depending on surface composition and impacting ion (Benninghoven et al. 1987). Elphic et al. (1991) analyzed lunar soil simulants and derived absolute ion sputter yields for solar wind sputtering conditions for many species in the range of $2 \times 10^{-7}$ to $2 \times 10^{-5}$. Considering the element composition of these soils, we obtain relative ion sputter yields that

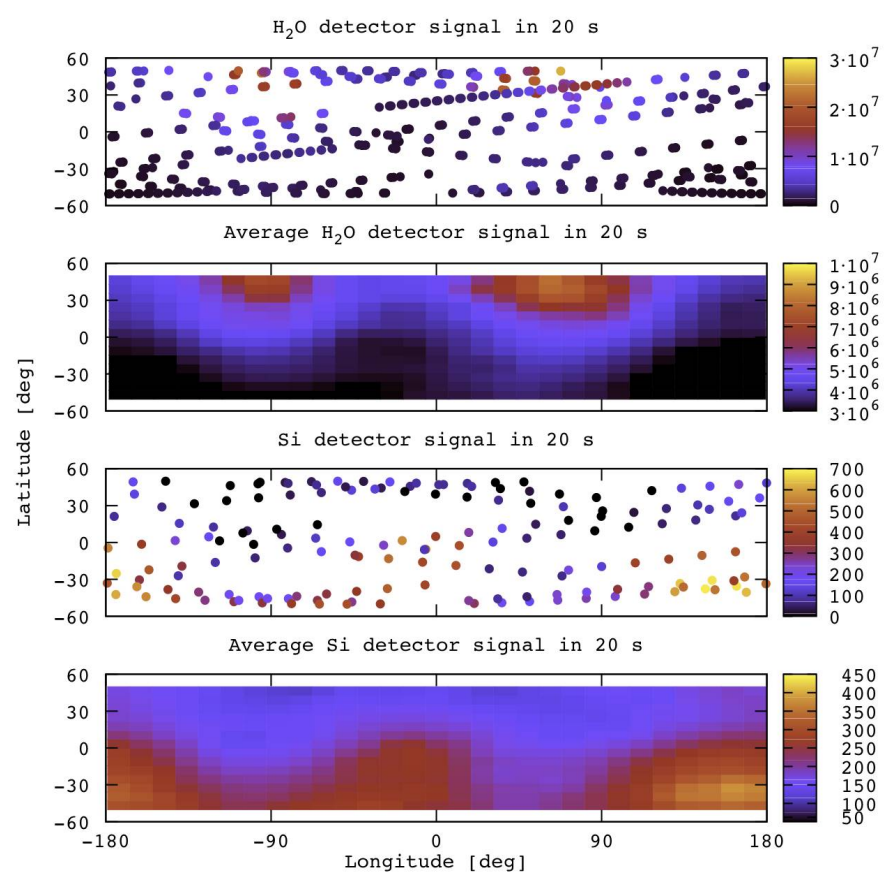

Fig. 3. Maps for the $\mathrm{H}_{2} \mathrm{O}$ (top two panels) and the Si signal (bottom two panels) measured with DFMS. Top panel and third panel from the top: actual measurements, normalized onto a $10 \mathrm{~km}$ sphere around the nucleus. Other two panels: interpolation of these signals to a complete map.

are between 10 and 100 times lower than the neutral sputter yields. Furthermore, sputtered ions might charge exchange with the neutral gas of the coma, reducing the amount of sputtered ions in the sputtered flux. Given the other uncertainties in our measurement, we did not further consider sputtered ions in our analysis.

DFMS is very restrictive in terms of field of view and energy for ions, and therefore we were only able to detect a small fraction of the distribution function of ions. At this time, we can only see the major volatiles as ions (Fuselier et al. 2015). DFMS will be much better suited to observe ions when Rosetta is inside a diamagnetic cavity, once it formed, when the plasma is cold and more or less follows the neutral gas. This will most likely be a difficult time to observe sputtered elements, however.

Pick-up ions (PUI) were observed during these times in the coma (Nilsson et al. 2015; Fuselier et al. 2015; Broiles et al. 2015), which might contribute to the sputter yield. However, these PUI first have to be accelerated to $\mathrm{keV}$ energies to cause significant sputtering by gyrating back to the cometary surface. Since their gyroradii are very large compared to the size of the comet, only a negligibly fraction will actually hit the comet. 


\subsection{Projection onto longitude/latitude map}

The data of the sputtered signal were projected onto the subspacecraft footpoint and were normalized to a $10 \mathrm{~km}$ sphere around the comet (see Fig. 3). Sputtered atoms can reach the spacecraft from many locations on the surface. Therefore one has to be cautious when interpreting the obtained maps because the projection of the measurements to the sub-spacecraft location on the cometary surface is a gross simplification of the actual source regions of the sputtered atoms measured with DFMS. Still, projections show clear differences between the sputtered atoms and the water, $\mathrm{H}_{2} \mathrm{O}$, the major volatile species in the coma of the comet in the northern hemisphere. Water outgassing peaks at times when the spacecraft is located above the summer hemisphere (positive latitudes) when the largest cross section of the comet is exposed to the Sun. This is the case when the subspacecraft point is above the neck region of the comet, that is, around $-90^{\circ}$ and $+60^{\circ}$ longitude in the Cheops coordinate system (Jorda et al. 2015). This is shown in Fig. 3 together with the distribution of silicon, the major identified sputtered component observed by ROSINA.

Our surface maps were interpolated from the individual measurements normalized to a $10 \mathrm{~km}$ sphere (see Fig. 3). The middle plate of Fig. 3 shows the corresponding individual measurements of silicon before interpolation. The sputtered silicon shows a behavior distinctly different from $\mathrm{H}_{2} \mathrm{O}$, it is mostly observed in areas of low water activity. Therefore, the highest sputter fluxes are observed when the spacecraft is above the less illuminated winter hemisphere and above either of the two lobes, that is to say, when the smallest cross section of the comet is exposed to the Sun and in the field of view of DFMS.

\subsection{Model for the absorption of solar wind protons}

In addition to the solar wind density and velocity, the interaction of the solar wind ions with the neutral gas coma has to be taken into account as well. Nilsson et al. (2015) reported that a non-negligible fraction of the solar wind ions interact with the neutral gas coma through charge-exchange reactions. This is of particular interest here as well: Fig. 3 shows a clear decrease in the signal of sputtered refractory species in the summer hemisphere of the comet. This hemisphere shows much more activity that is due to the higher solar insolation (Bieler et al. 2015). Both the measured abundances of volatiles and the sputtered species are expected to correlate with the geometric cross section of the comet exposed to the Sun, that is, the total surface under solar insolation (volatiles) or bombardment of solar wind ions (sputtered products). The second dependency is the cross section of these areas in the field of view of ROSINA. The details can be found in Bieler et al. (2015).

Since a high abundance of volatiles prevents the solar wind from reaching the surface, which we elaborate on below, the peak in the sputtered products is located above the two lobes of the comet and does not occur at times when the exposed cross sections are largest. This location is clearly different from the volatile species observed by ROSINA, as can be seen in Fig. 3 by comparing the $\mathrm{H}_{2} \mathrm{O}$ and the $\mathrm{Si}$ signals.

In the following we calculate the fraction of solar wind ions that reach the surface of the comet. We also consider collisions between the sputtered atoms, which further distributes them in the coma through scattering. COPS data and 3D coma modelling (the 3D coma model is described in Tenishev et al. 2008, 2011; Bieler et al. 2015) were used to derive an approximate column density of neutral gas atoms and molecules between the

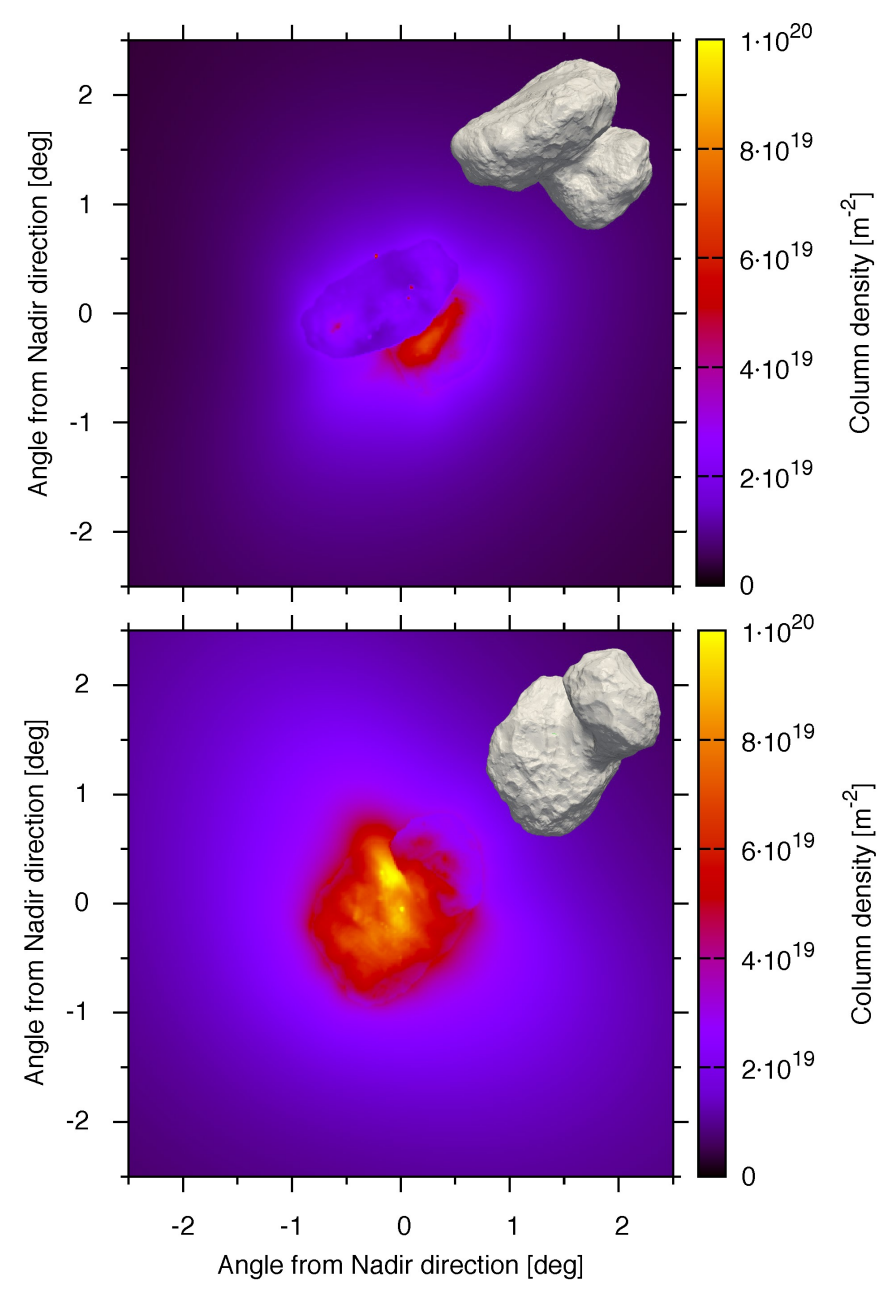

Fig. 4. Modelled column densities of water for two different situations of the comet exposed to the Sun as seen from the Sun direction at a distance of $130 \mathrm{~km}$, for low (top panel) and high (bottom panel) calculated column densities (Bieler et al. 2015). Insets: approximate orientation of the comet in these calculations.

spacecraft and the comet through which the solar wind has to pass before reaching the surface. From ROSINA observations only the column along the comet spacecraft direction can be estimated (red dashed and blue arrows in Fig. 1). The column density along the red solid arrow, that is, the column that has to be passed by the solar wind, is estimated by multiplying the column density along the red dashed direction, which is derived directly from the COPS measurement by a calibration factor of 6 obtained by comparison to the Monte Carlo calculations presented in Fig. 4. Our simplified model calculates the neutral gas column density the solar wind has to pass, $N_{\mathrm{sw}}$, from infinity to the surface of the comet with radius $r_{\mathrm{c}}$, by applying a $1 / r^{2}$ law using the scaled number density of COPS for the subsolar point, $n_{\mathrm{R}}$, at the distance of the Rosetta spacecraft, $r_{\mathrm{R}}$, from the comet,

$N_{\mathrm{sw}}=\int_{r_{\mathrm{c}}}^{\infty} n_{\mathrm{R}} \frac{r_{\mathrm{R}}^{2}}{r^{2}} \mathrm{~d} r=n_{r} \frac{r_{\mathrm{R}}^{2}}{r_{\mathrm{c}}}$.

The ratio of solar wind ions arriving at the surface of the comet with energies high enough to sputter particles, $f$, compared to the total amount of ions, $f_{0}$, is

$\frac{f}{f_{0}}=\exp \left(-N_{\mathrm{sw}} \sigma\right)$ 
Equation (5) is derived by the assumption that the amount of solar wind ions interacting with the neutral cloud simply depends on the local neutral gas density, $n(r)$, and the scattering cross section, $\sigma$. Since the charge exchange cross section is significantly larger than the scatter cross section, many solar wind ions suffer charge exchange with the neutral gas of the coma and arrive at the nucleus as neutral atoms, which has no effect on the sputter yield (Wurz et al. 2007). Considerable numbers of PUI as a result of this charge exchange were observed in the plasma measurements (Nilsson et al. 2015). For the fraction of solar wind ions that reach the surface, we solve

$\frac{\mathrm{d}}{\mathrm{d} r} f(r)=n(r) \sigma f(r)$,

with the density at distance, $r$, from the center of the nucleus as discussed above,

$n(r)=n_{\mathrm{R}} \frac{r_{\mathrm{R}}^{2}}{r^{2}}$

For the scattering cross section we have assumed $\sigma=3 \AA^{2}$. Collisions between solar wind ions with the cometary gas will be mostly close to the comet because of the density profile of cometary gas. Because of the mismatch of masses between the protons or hydrogen atoms and water molecules, the solar wind flux to the surface will mostly be reduced by angular scattering and not by energy loss. Since several scattering events are necessary to significantly deflect solar wind particles from reaching the surface, we used a cross section value that compounds the effect of several collisions. For the radius of the comet we used $r_{\mathrm{c}}=2 \mathrm{~km}$. We then compared the obtained column densities with the above-mentioned global 3D model to derive the calibration factor needed to relate column densities of the red dashed arrow and the red solid arrow from Fig. 1. Two examples of these simulations are shown in Fig. 4 for a large and small cross section of the comet exposed to the Sun. The global simulations and the COPS measurements agree on the locations where they can be compared (Bieler et al. 2015).

The same approach was used for the fraction of the sputtered atoms from the surface reaching the Rosetta spacecraft,

$\frac{f}{f_{0}}=\exp \left(-N_{\mathrm{sp}} \sigma\right)$

with

$N_{\mathrm{sp}}=\int_{r_{\mathrm{c}}}^{r_{r}} n_{\mathrm{R}} \frac{r_{\mathrm{R}}^{2}}{r^{2}} \mathrm{~d} r=n_{\mathrm{r}} \frac{r_{\mathrm{R}}\left(r_{\mathrm{R}}-r_{\mathrm{c}}\right)}{r_{\mathrm{c}}}$.

This simple model calculation shows that the estimate for the transmission matches the observations well: the high neutral gas column density, $N_{\text {sw }}$ anticorrelates with the sputtered $\mathrm{Si}$ signal. The water column density needed to attenuate the solar wind flux to the $1 / \mathrm{e}$ level is approximately $N_{\mathrm{SW}}=3 \times 10^{16} \mathrm{~cm}^{-2}$. Of course this model is only an approximation. The comet does not outgas uniformly over the surface as shown in the data presented above (see Fig. 3) and in earlier works (Hässig et al. 2015), nor is the comet a sphere. However, this calculation was used to identify regions on the maps that are minimally influenced by the volatile species of the coma.

Since Rosetta flew in the terminator plane in bound orbits for the time period of these measurements, it is expected that the actual column of gas is higher on the sunward side of the comet, and our calibration factor of 6 is only an approximation. Nevertheless, most solar wind ions and sputtered atoms are lost

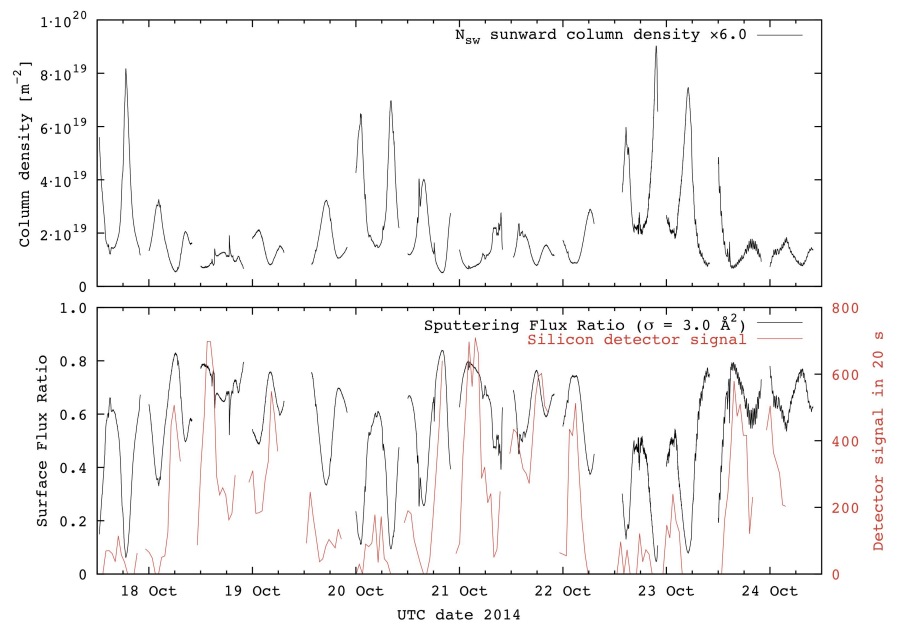

Fig. 5. Top panel: column density $N_{s w}$ derived from COPS density measurements (using Eq. (4)), adjusted to the direction of solar wind flow. Bottom panel: calculated flux ratio for solar wind particles that reach the cometary surface and sputtered atoms back to the ROSINA observation point (black lines), using Eq. (5). The sputtered Si signal is shown as red lines. Interruptions in the lines were caused at times when the sensors were turned off.

in the last few kilometers above the surface, the region that is increasingly dominated by the local gas production rate on the nucleus. Furthermore, the assumed scattering cross section of $3 \AA^{2}$ is not very well known. More detailed modelling is required, but we defer this part of the work to a future investigation.

The sputtered signal clearly shows an anticorrelation with the observed total neutral gas density from the COPS. Figure 5 shows the result of this calculation. We note the good correlation between the transmitted flux and the sputtered Si signal.

Dust grains released from the surface of the comet are also sputtered by the solar wind that contributes to the signal. However, because of the low activity of the comet, the dust emission is also low (Rotundi et al. 2015), with an estimated dust grain density of $0.1 \mathrm{~m}^{-3}$ at the spacecraft location of $10 \mathrm{~km}$ above the sun-lit surface, thus their contribution to the sputter signal is considered negligible. Moreover, dust particles are set free from the comet by gas drag, thus they are released preferentially from the sun-lit surface, that is, from the summer hemisphere; in the winter hemisphere dust densities will be even lower there and the contribution to our signal is even lower, contrary to our observation of an anticorrelation between water and the sputtered signal.

\section{Results}

Both our model and the observations show increased sputtering on the winter hemisphere, which is considerably less active for $\mathrm{H}_{2} \mathrm{O}$ than in the summer hemisphere. The ratio of sputtered elements from both hemispheres is roughly one-third. We therefore present our results when using all available data and when considering only measurements obtained above and below $0^{\circ}$ latitude, the latter representing the winter hemisphere with increased sputtering and therefore better signal-to-noise ratio. Table 2 presents the results side-by-side and shows that they are the same to within a few percent.

Silicon, ${ }^{28} \mathrm{Si}$, represents the most dominant sputtered species observed by ROSINA DFMS. Similar amounts of other refractory elements common to mineral grains might be expected, such as magnesium, ${ }^{24} \mathrm{Mg}$, and iron, ${ }^{56} \mathrm{Fe}$. However, these species 
P. Wurz et al.: Sputtering of cometary dust

Table 2. Derived elemental ratios for 67P from the sputtered signals, averaged over the winter hemisphere or averaged over all measurements.

\begin{tabular}{cccccl}
\hline \hline Ratio & Both hemispheres & Winter hemisphere & Summer hemisphere & CI & CV \\
\hline $\mathrm{Na} / \mathrm{Si}$ & 0.021 & 0.019 & 0.024 & 0.05747 & 0.0265 \\
$\mathrm{Ca} / \mathrm{Si}$ & 0.016 & 0.014 & 0.019 & 0.05968 & 0.0821 \\
$\mathrm{~K} / \mathrm{Si}$ & 0.0022 & 0.0022 & 0.0022 & 0.003582 & 0.00165 \\
\hline
\end{tabular}

Notes. Uncertainties in the ratios are $30 \%$. The carbonaceous chondrite (CI) values are adopted from Lodders (2003), the carbonaceous chondrite (CV) values from McSween \& Huss (2010).

are rather hard to detect because of the lower detection efficiencies for higher mass species such as ${ }^{56} \mathrm{Fe}$, lower ionization cross sections, about a factor 2 for ${ }^{24} \mathrm{Mg}$ compared to ${ }^{28} \mathrm{Si}$ (Vainshtein et al. 1972), for example, and most importantly, because of interferences. ${ }^{24} \mathrm{Mg}(23.9845 \mathrm{u} / \mathrm{e})$, for instance, is overlapped by doubly charged sulphur oxide, $\mathrm{SO}^{++}(23.9829 \mathrm{u} / \mathrm{e})$, a fragment of cometary sulphur dioxide, $\mathrm{SO}_{2}$, created inside DFMS' ion source. Sulphur-bearing species are quite abundant in the coma and produce sizeable amounts of doubly charged ions in the electron impact ionization process (Le Roy et al. 2015). Moreover, ${ }^{56} \mathrm{Fe}$ will interfere with $\mathrm{CaO}$ if it were sputtered by ion impact. Sodium, ${ }^{23} \mathrm{Na}(22.9892 \mathrm{u} / \mathrm{e})$, can be separated well (see Fig. 2) from the doubly charged carbon dioxide peak with the heavy oxygen isotope, $\mathrm{C}^{16} \mathrm{O}^{18} \mathrm{O}^{++}(22.9965 \mathrm{u} / \mathrm{e})$. ${ }^{40} \mathrm{Ca}(39.9620 \mathrm{u} / \mathrm{e})$ can also be separated from a potential $\mathrm{S}_{2} \mathrm{O}^{++}$ (39.9690 u/e), which is again a doubly charged sulphur-bearing component. ${ }^{40} \mathrm{Ar}$ is not expected to be present in significant amounts. However, the main difficulty arises from the low count rates of ${ }^{40} \mathrm{Ca}$ and more so for the low counts rates of ${ }^{39} \mathrm{~K}$ $(38.9632 \mathrm{u} / \mathrm{e})$. Both of these species are not present in all spectra, but still show an enhancement on the winter hemisphere when averaging over all available datasets.

There are several other species of interest for studying the inventory of refractory elements of the comet. Unfortunately, the faint ${ }^{24} \mathrm{Mg}$ (23.9845 u/e) peak sits at the shoulder of the strong $\mathrm{C}_{2}$ peak; similarly, the other magnesium isotopes are masked by $\mathrm{C}_{2} \mathrm{H}$, and $\mathrm{C}_{2} \mathrm{H}_{2}$, respectively. ${ }^{27} \mathrm{Al}$ is masked by the strong $\mathrm{HCN}$ peak, and ${ }^{48} \mathrm{Ti}$ is masked by the very strong SO peak. We also have maps of sulphur, but $\mathrm{S}$ most likely is a fragment of sulphurbearing volatile components, which are far more abundant, and therefore mask the sputtered sulphur peak. The same applies to oxygen and carbon.

\subsection{Average composition}

From the observations presented in Fig. 6, we calculated averages of elemental ratios over large areas of the surface of the comet: the areas being the winter and the summer hemisphere and both hemispheres together. The data are presented in Table 2 together with composition data from $\mathrm{CI}$ and $\mathrm{CV}$ carbonaceous chondrites (Lodders 2003; McSween \& Huss 2010). CIs are considered to be chemically the most primitive meteorites, and $\mathrm{CV}$ s are very primitive as well and have a very low content of volatiles. The winter hemisphere is considered the best data set from this analysis because the influence of volatile material in the coma is the smallest.

$\mathrm{The} \mathrm{Na} / \mathrm{Si}$ of the comet is compatible with the $\mathrm{CV}$ value (if we consider only the winter hemisphere, the values are almost identical), but is less than half of the CI value. For comparison, in a significantly higher $\mathrm{Na} / \mathrm{Si}$ ratio of 0.054 was found for dust collected in the coma at perihelion in comet Halley (Jessberger et al. 1988).

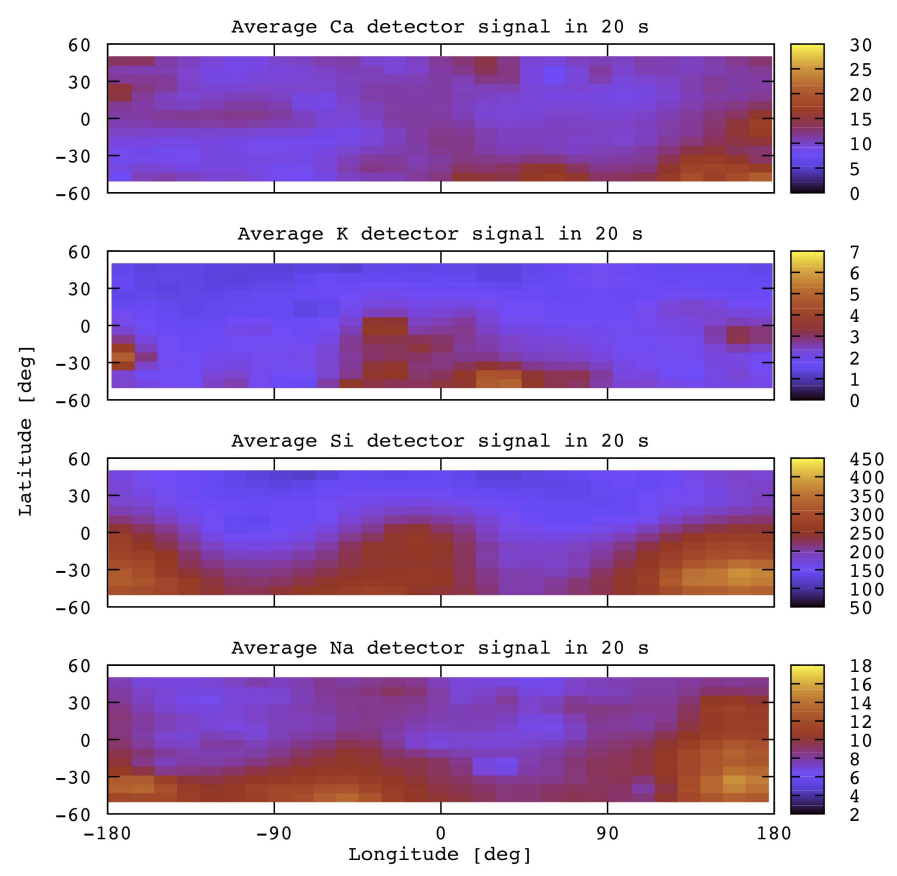

Fig. 6. Maps for the sputtered signal of $\mathrm{K}, \mathrm{Si}, \mathrm{Ca}$, and $\mathrm{Na}$ measured with DFMS.

The $\mathrm{Ca} / \mathrm{Si}$ ratio of the comet is significantly lower than the $\mathrm{CI}$ and the $\mathrm{CV}$ value. Considering the winter hemisphere value, the $\mathrm{Ca}$ depletion is by about a factor of five compared to $\mathrm{CV}$ and a factor three compared to $\mathrm{CI}$. For comparison, a $\mathrm{Ca} / \mathrm{Si}$ ratio of 0.034 was found in comet Halley (Jessberger et al. 1988).

The $\mathrm{K} / \mathrm{Si}$ ratio is somewhat higher than the $\mathrm{CV}$ value, by about $40 \%$, but lower than the CI value by about $60 \%$. For comparison, a K/Si ratio of 0.0011 was found in comet Halley (Jessberger et al. 1988).

In summary, we found that the composition of 67P does not match the CI or the CV chondrite values for the studied species. $\mathrm{Na}$ abundance is lower than $\mathrm{CI}$, but compatible with $\mathrm{CV}, \mathrm{Ca}$ is heavily depleted by a factor of 3 to 5 compared to CI and CV values, respectively, and $\mathrm{K}$ is found to be between the $\mathrm{CI}$ and the $\mathrm{CV}$ values. Given that all elemental ratios we found are lower than the CI values, the $\mathrm{Si}$ abundance is likely higher than the $\mathrm{CI}$ chondritic value. We note that depletions in $\mathrm{Ca}$ and $\mathrm{Mg}$ are observed for interplanetary dust particles (IDPs; Schramm et al. 1989), most pronounced for smooth dust particles (CS type) and less so for the porous dust particles (CP type). Depletion in $\mathrm{Ca}$ and $\mathrm{Mg}$ in IDPs was explained by aqueous alteration, which belongs to the earliest and most widespread geological processes in the solar system, occurring within the first tens of million of years of solar system history.

Trigo-Rodíguez \& Llorca (2007) investigated cometary meteoroids burning up in the terrestrial atmosphere by spectroscopy 
Table 3. Sodium-to-potassium ratios in the solar system.

\begin{tabular}{ccc}
\hline \hline Object & $\mathrm{Na} / \mathrm{K}$ & Source \\
\hline Mercury surface & 24 & Evans et al. (2012), Peplowski et al. (2012) \\
Lunar crust & $7-9$ & Lodders \& Fegley (1998, p. 177) \\
Meteorites & $7-9$ & Lodders \& Fegley (1998, p. 311) \\
Earth atmosphere & $20-150$ & Gault \& Rundle (1969) \\
Earth seawater & 27 & Lodders \& Fegley (1998, p. 164) \\
Earth crust & 2 & Lodders \& Fegley (1998, p. 143) \\
Solar & 15.5 & Asplund et al. (2009) \\
Solar system & 15 & Lodders \& Fegley (1998, p. 80) \\
67P/ C-G Both hemispheres & 9.5 & This work \\
\hline
\end{tabular}

Notes. Uncertainties in our ratios are 30\%. The value for Mercury's surface is derived from the average Na measurement reported in Evans et al. (2012) and the average K value reported in Peplowsky et al. (2012).

methods. They found a $\mathrm{Na} / \mathrm{Si}$ ratio in the range of 0.04 to 0.1 , with all measurements higher than the chondritic value. The authors suggested that $\mathrm{Na}$ is enhanced in these cometary meteoroids on average by a factor of 1.5 to the chondritic value.

Schulz et al. (2015) investigated individual dust grains set free from the comet and collected with the COSIMA instrument. They reported element ratios up to $\mathrm{Na} / \mathrm{Mg}=0.8$, which they interpreted as the dust grains being generally rich in sodium. Since we did not measure $\mathrm{Mg}$, we cannot directly compare with a global value. However, we found that $\mathrm{Na}$ is nearly chondritic (CV), thus the $\mathrm{Mg}$ abundance must be very low, assuming the COSIMA Na/Mg measurement can be generalized as a global value. A low $\mathrm{Mg}$ value in the cometary dust would fit well with DFMS not detecting $\mathrm{Mg}$ above the level of $\mathrm{SO}^{++}$.

$\mathrm{Na}$ and $\mathrm{K}$ are two moderately volatile elements and can give clues about the processes these grains might have suffered during their life. Thus, we also calculated $\mathrm{Na} / \mathrm{K}$ ratios from our measurements, which are shown in Table 3 together with data for other solar system objects. Considering our uncertainties, the $\mathrm{Na} / \mathrm{K}$ value for $67 \mathrm{P}$ is compatible with the meteoritic values, the solar, and the solar system value. This is different to comet Halley, where a $\mathrm{Na} / \mathrm{K}=50$ was found for the dust in the coma near perihelion (Jessberger et al. 1988). Furthermore, Fulle et al. (2013) determined this ratio for the comet C/2011 L4 (Panstarrs) near perihelion by spectroscopy methods as $\mathrm{Na} / \mathrm{K}=54 \pm 14$, which was considered compatible with the solar value after accounting for the details of the observation.

\subsection{Heterogeneity}

Silicon is the element for which we have the best statistics, and it is the only species we can use to discuss possible features of chemical heterogeneity of refractory material on the surface (Fig. 6). Figure 3 shows that most of the heterogeneity seen in the Si maps is due to the varying absorption of solar wind caused by the variations in the water column density. A local enhancement at longitudes of $+110^{\circ}$ to $+180^{\circ}$ to $-150^{\circ}$ longitude remains on the body of the comet, where the intensity is increased by about $30 \%$ at most. Even for this feature, solar wind reduction by the water column in the coma may be the reason.

\section{Conclusions}

The fortuitous combination of the Rosetta spacecraft being very close to the comet during a period of low activity allows the observation of sputtered refractory elements from the surface of the comet. This observation was not scheduled. We derived global, or semi-global, values for a few element ratios. These global values are complementary to measurements on the level of individual grains to be made by COSIMA.

Acknowledgements. The authors would like to thank the following institutions and agencies, which supported this work: Work at UoB was funded by the State of Bern, the Swiss National Science Foundation and by the European Space Agency PRODEX Program. Work at MPS was funded by the Max-Planck Society and BMWI under contract 50QP1302. Work at Southwest Research institute was supported by subcontract \#1496541 from the Jet Propulsion Laboratory. Work at BIRA-IASB was supported by the Belgian Science Policy Office via PRODEX/ROSINA PEA 90020. This work was supported by CNES grants at IRAP, LATMOS, LPC2E, UTINAM, CRPG. Work at the University of Michigan was funded by NASA under contract JPL-1266313. ROSINA would not give such outstanding results without the work of the many engineers, technicians, and scientists involved in the mission, in the Rosetta spacecraft, and in the ROSINA instrument team over the last 20 years, whose contributions are gratefully acknowledged. We gratefully acknowledge the work of the whole ESA Rosetta team. Rosetta is an ESA mission with contributions from its member states and NASA.

\section{References}

Asplund, M., Grevesse, N. A., Sauval, J., \& Scott, P. 2009, ARA\&A, 47, 481 Balsiger, H., Altwegg, K., Bochsler, P., et al. 2007, Space Sci. Rev., 128, 745 Benninghoven, A., Rüdenauer, F. G., \& Werner, H. W. 1987, in Secondary Ion Mass Spectrometry, Chemical Analysis (Wiley), 86

Bieler, A., Altwegg, K., Balsiger, H., et al. 2015, A\&A, 583, A7

Biersack, J. P., \& Eckstein, W. 1984, Appl. Phys. A, 34, 73

Borovik, A. A. 2012, Techn. Phys., 57, 1058

Broiles, T. W., Burch, J. L. Clark, G., et al. 2015, A\&A, 583, A21

Eckstein, W., \& Urbassek H. M. 2007, in Computer simulation of the sputtering process, in Sputtering by particle bombardment: experiments and computer calculations from threshold to MeV energies, eds. R. Behrisch, \& W. Eckstein (Berlin: Springer), 21

Elphic, R. C., Funsten III, H. O., Barraclough, B. L., et al. 1991, Geophys. Res. Lett., 18, 2165

Evans, L. G., Peplowski, P. N., Rhodes, E. A., et al. 2012, J. Geophys. Res., 117, 00L07

Freund, R. S., Wetzel, R. C., Shul, R. J., \& Hayes, T. R. 1990, Phys. Rev. A., 41, 3575

Fujii, K., \& Srivastava, S. K. 1995, J. Phys. B: At., Mol. Opt. Phys., 28, L559

Fulle, M., Molaro, P., Buzzi, L., \& Valisa, P. 2013, ApJ, 771, L1

Fuselier, S. A., Altwegg, K., Balsiger, H., et al. 2015, A\&A, 583, A2

Gault, W. A., \& Rundle, H. N. 1969, Can. J. Phys., 47, 85

Gombosi, T. 2015, Physics of Cometary Magnetospheres, in Magnetotails in the Solar System, eds. A. Keiling, C. M. Jackman, \& P. A. Delamere, Geophysical Monograph 207 (American Geophysical Union), 169

Hässig, M., Altwegg, K., Balsiger, H., et al. 2015, Science, 347, 0276

Haynes, W. M. 2013, CRC Handbook of chemistry and physics: a readyreference book of chemical and physical data (CRC Press)

Hijazi, H., Bannister, M. E., Meyer III, H. M., Rouleau, C. M., et al. 2014, J. Geophys. Res. Sp. Phys., 119, 8006

Jessberger, E. K., Christoforidis, A., \& Kissel, J. 1988, Nature, 332, 691 
P. Wurz et al.: Sputtering of cometary dust

Jorda, L., Gaskell, R, Hviid, S. F, et al, 2015, RO-C-OSINAC/OSIWAC-567P-SHAPE-V1.0. NASA Planetary Data System and ESA Planetary Science Archive

Le Roy, L., Altwegg, K., Balsiger, H., et al. 2015, A\&A, 583, A1

Lodders, K. 2003, ApJ, 591, 1220

Lodders, K., \& Fegley, B. 1998, The Planetary Scientists Companion (Oxford University Press)

McSween, H., \& Huss, G. 2010, Cosmochemistry, 393

Meier, R., \& Eberhardt, P. 1993, Int. J. Mass Spectr., 123, 19

Nilsson, H., Stenberg Wieser, G., Behar, E., et al. 2015, Science, 347, 0571

Peplowski, P. N., Lawrence, D. J., Rhodes, E. A., et al. 2012, J. Geophys. Res., 117, E00L04

Rotundi, A., Sierks, H., Della Corte, V., et al. 2015, Science, 347, 3905-1

Schramm, L. S., Brownlee, D. E., \& Wheelock, M. M. 1989, Meteoritics, 24 99
Schulz, R., Hilchenbach, M., Langevin, Y., et al. 2015, Nature, 518, 216

Tenishev, V., Combi, M., \& Davidsson, B. 2008, ApJ, 685, 659

Tenishev, V., Combi, M. R., \& Rubin, M. 2011, ApJ, 732, 104

Trigo-Rodríguez, J. M., \& Llorca, J., 2007, Adv. Sp. Res., 39, 517

Vainshtein, L A., Ochkur, V. I., Rakhovskii, V. I., \& Stepanov, A. M. 1972, Sov. J. Exp. Theo. Phys., 34, 271

Wehner, G., Kenknight, C., \& Rosenberg, D. L. 1963, Planet. Space Sci., 11, 885 Wurz, P. 2012, in Nano Dust in the Solar System: Discoveries and Interpretations, Astrophys. Space Sci. Lib., 385, 161

Wurz, P., Rohner, U., Whitby, J. A., et al. 2007, Icarus 191, 486

Wurz, P., Whitby, J. A., Rohner, U., et al. 2010, Planet. Space Sci., 58, 1599

Ziegler, J. F. 2004, Nucl. Instr. Meth. B, 219, 1027

Ziegler, J. F., Biersack, J. P., \& Littmark, U. 1984, The Stopping and Range of Ions in Solids, Vol. 1 of series Stopping and Ranges of Ions in Matter (New York: Pergamon Press) 